\title{
DETC2010-28431
}

\section{VALIDATING DESIGNS THROUGH SEQUENTIAL SIMULATION-BASED OPTIMIZATION}

\author{
Jing Li \\ Mechanical Engineering Department \\ Oakland University, Rochester, MI 48309, USA \\ Email: li2@oakland.edu
}

\author{
Michael Kokkolaras \\ Department of Mechanical Engineering \\ University of Michigan, Ann Arbor, Ml 48109, USA \\ Email:mk@umich.edu
}

\author{
Zissimos P. Mourelatos \\ Mechanical Engineering Department \\ Oakland University, Rochester, MI 48309, USA \\ Email: mourelat@oakland.edu
}

\author{
Panos Y. Papalambros \\ Department of Mechanical Engineering \\ University of Michigan, Ann Arbor, MI 48109, USA \\ Email: pyp@umich.edu
}

\author{
David J. Gorsich \\ US Army, TARDEC \\ Warren, MI, USA \\ E-mail: david.gorsich@us.army.mil
}

\begin{abstract}
Computational simulation models support a rapid design process. Given model approximation and operating conditions uncertainty, designers must have confidence that the designs obtained using simulations will perform as expected. This paper presents a methodology for validating designs as they are generated during a simulation-based optimization process. Current practice focuses on validation of simulation models throughout the entire design space. In contrast, the proposed methodology requires validation only at design points generated during optimization. The goal of such validation is confidence in the resulting design rather than in the underlying simulation model. The proposed methodology is illustrated on a simple cantilever beam design subject to vibration.
\end{abstract}

\section{INTRODUCTION}

Design optimization often requires computational analysis or simulation models. These models quantify functional inputoutput relations contained in the objective and constraints. Such models are inexact approximations of the physical world, and so we need to quantify our confidence that designs obtained using simulations will perform as expected when produced. Current practice uses computational models for optimization studies in relatively large design spaces even though the models have been validated only in a small subset of the design space. Within this paradigm, computational models will need to be validated for the entire feasible design space in order to obtain high confidence in the results.

The motivation for the present work is that the aforementioned global model validation may not be necessary. A numerical optimization process creates a sequence of design iterates, whose validity is important only at the optimum. One way to concentrate on the validity of the optimal design rather than that of the model, is to conduct validation studies at the design iterates as they are generated.

In this paper, we adopt the idea of a sequential optimization approach where the design space at each iteration is much smaller than the entire feasible space. Starting with a validated initial design we conduct optimization within a limited design space around that design. If the optimization outcome, i.e., the next design iterate, lies on the boundary of the smaller design space*, we examine its validity; if it is acceptable, we start a new optimization; otherwise, we calibrate the model so that its validity is acceptable at that new

\footnotetext{
* The design space is defined by the bounds of the design optimization variables, while the feasible space is defined by the intersection of all design constraints.
} 
design iterate. If the model cannot be calibrated to acceptable validity levels, the designer may want to abort the sequential optimization process until a more accurate model becomes available, or continue while being aware of the model's limitation. If the new design iterate lies in the interior of the design space of the last optimization, we have converged to an optimal and validated design. This approach can be also applied to parametric studies to ensure that a design is valid for different operating conditions.

The surrogate management framework by Booker et al. [1] offers a variation of the main idea presented and implemented here. Booker et al. generate a sequence of calibrated approximations (metamodels or surrogate models) of the objective function only, which they manage for direct surrogate optimization. They do not assess the validity of their approximations; they simply improve it by calibration during optimization. In the present work, we assess the validity of the design iterates generated by sequential optimization in subsets of the design space by validating the simulation model at these subsets and improving it only if necessary, provided testing can be performed at these points.

The paper is organized as follows. Section 2 describes the proposed approach. Section 3 provides an overview of the confidence level quantification including a brief description of the Probabilistic Principal Component Analysis (PPCA) and the interval-based Bayesian hypothesis testing method. Section 4 uses the vibratory response of a cantilever beam to illustrate the steps of the methodology, and Section 5 presents a summary and conclusions.

\section{PROPOSED METHOD}

Let the $n$-dimensional domain $D$ consist of all designs $\mathbf{d} \in \mathfrak{R}^{n} \quad$ such that $D=\{\mathbf{d}: \underline{\mathbf{d}} \leq \mathbf{d} \leq \overline{\mathbf{d}}\}$ with $\underline{\mathbf{d}}$ and $\overline{\mathbf{d}}$ denoting lower and upper design variable bounds, respectively. Local domains $D_{j}, j=1, \ldots, J$ are defined so that $D_{j}$ is a proper subset of $D\left(D_{j} \subset D\right)$ with $D_{j}=\left\{\mathbf{d}: \underline{\mathbf{d}}^{j} \leq \mathbf{d} \leq \overline{\mathbf{d}}^{j}\right\}$ and $\underline{\mathbf{d}} \leq \underline{\mathbf{d}}^{j} \leq \overline{\mathbf{d}}^{j} \leq \overline{\mathbf{d}}$. Two local domains $D_{j}$ and $D_{j+1}$ may overlap $\left(D_{j} \cap D_{j+1} \neq 0\right)$ or may be disjoint $\left(D_{j} \cap D_{j+1}=0\right)$. Figure 1 shows two overlapping local domains in two dimensions.

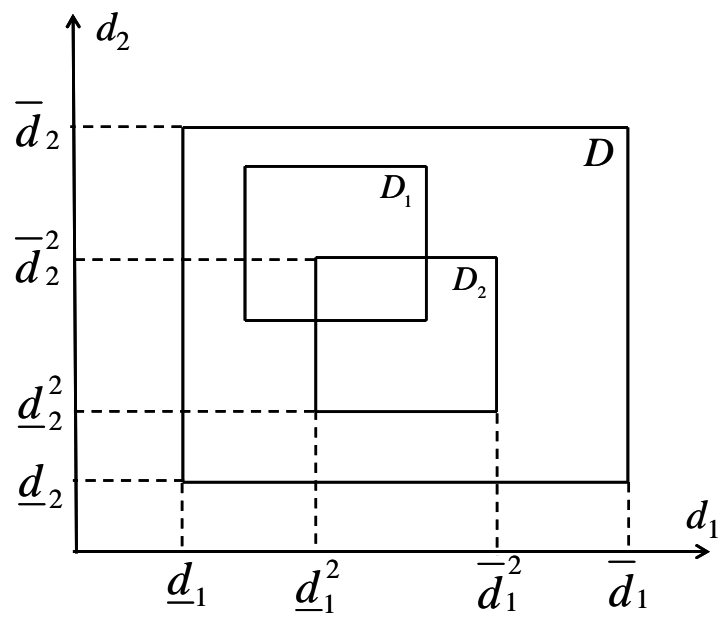

Figure 1. Notation and definition of local domains
All designs $\mathbf{d}$ in domain $D$ are considered valid if

$$
\min _{\mathbf{d} \in D} C(\hat{\mathbf{y}}(\mathbf{d}, \mathbf{p}), \mathbf{y}(\mathbf{d})) \geq C^{t}
$$

where $C$ quantifies our confidence that the model prediction $\hat{\mathbf{y}}(\mathbf{d}, \mathbf{p})$ is statistically close to the true function value $\mathbf{y}(\mathbf{d})$. The former is obtained using a computational model (CAE), and the latter by testing. In Equation (1), $C^{t}$ is the target confidence value. Note that the CAE prediction $\hat{\mathbf{y}}(\mathbf{d}, \mathbf{p})$ depends not only on $\mathbf{d}$, but also on a vector of calibration parameters $\mathbf{p}$. If for a design $\mathbf{d}$ the confidence value is low, we assume that the calibration parameters $\mathbf{p}$ can be adjusted to increase it.

Now we answer the following question. If a design $\mathbf{d} \in D$ is valid, but Equation (1) cannot be satisfied for every design in $D$, can we identify a sub-domain $D_{s} \subset D$ such that $\mathbf{d} \in D_{s}$ and $\min _{\mathbf{d}_{t} \in D_{s}} C(\hat{\mathbf{y}}(\mathbf{d}, \mathbf{p}), \mathbf{y}(\mathbf{d})) \geq C^{t}$ by adjusting $\mathbf{p}$ ? In other words, if there exists a valid design $\mathbf{d}$ in the domain $D$, can we identify a smaller domain $D_{s}$ which includes $\mathbf{d}$ for which Equation (1) holds? If this is true, the design will be valid in $D_{s}$ but it will not necessarily be valid in $D-D_{s}$.

$$
\text { Let } D \supset D_{1} \supset D_{2} \supset \cdots \supset D_{i} \supset \cdots \supset D_{n} \text { and } D_{i}-D_{i+1}=
$$

$d D \quad$ (very small). If $\quad C_{\min }(i)=$
$\min _{\mathbf{d} \in D_{i}} C(\hat{\mathbf{y}}(\mathbf{d}, \mathbf{p}), \mathbf{y}(\mathbf{d})), i=1,2, \ldots, n$, we then have $D_{s} \equiv D_{i+1}$ where $\left\{i: C_{\min }(i+1) \geq C^{t}\right.$ and $\left.C_{\min }(i)<C^{t}\right\}$. This process can be used to identify the smallest domain $D_{s}$ such that $\min _{\mathbf{d} \in D_{s}} C(\hat{\mathbf{y}}(\mathbf{d}, \mathbf{p}), \mathbf{y}(\mathbf{d}))=C^{t}$.

The above procedure will identify $D_{s}$, if it exists. This depends on $C^{t}$. The choice of $C^{t}$ is therefore, critical. We want it to be as small as possible in order to avoid excessive calibrations that require expensive tests, but we also want it to be large enough to guarantee that the designs in $D_{s}$ are valid so that the optimization yields a valid optimal design. This is a minimax problem.

We propose to use a local domain approach and calculate $C$ at its center. If $C \geq C^{t}$, then we accept the CAE model as locally valid and use it to solve the optimization problem in the local domain. In doing so, we assume that the CAE model is valid (i.e., Equation (1) holds) in the entire local domain. Otherwise, we calibrate the CAE model by solving the following problem

$$
\max _{\mathbf{p}} C\left(\hat{\mathbf{y}}\left(\mathbf{d}_{c}, \mathbf{p}\right), \mathbf{y}\left(\mathbf{d}_{c}\right)\right),
$$

where a set of calibration parameters $\mathbf{p}$ is determined in order to maximize $C$ at the center $\mathbf{d}_{c}$ of the local domain. The resulting calibrated CAE model is accepted even if $\max _{\mathbf{p}} C(\hat{\mathbf{y}}(\mathbf{d}, \mathbf{p}), \mathbf{y}(\mathbf{d}))<C^{t}$ because $\max _{\mathbf{p}} C(\hat{\mathbf{y}}(\mathbf{d}, \mathbf{p}), \mathbf{y}(\mathbf{d}))$ is the best attainable $C$ value based on the chosen set $\mathbf{p}$ of calibration parameters and the design $\mathbf{d}_{c}$. If $\max _{\mathbf{p}} C(\hat{\mathbf{y}}(\mathbf{d}, \mathbf{p}), \mathbf{y}(\mathbf{d}))$ is not 
acceptable, we must change the calibration set $\mathbf{p}$ or adopt a more accurate CAE model.

If the optimizer $\mathbf{d}_{i}^{*}$ found after an optimization within a local domain $D_{i}$ lies on the boundary of $D_{i}$, we i) validate the CAE model by solving the problem of Equation (2) with $\mathbf{d}_{c i}=\mathbf{d}_{i}^{*}$, ii) create a new local domain $D_{i+1}$ centered at $\mathbf{d}_{i}^{*}$, and iii) conduct a new optimization within $D_{i+1}$. This sequential optimization process using local domains is repeated until the optimum is in the interior of the local domain. We emphasize that the local domain is defined by the bounds of the design variables only, i.e., it is not identical to the feasible space of the original optimization problem.

Note that by reducing the size of the local domain, we guarantee that a validated local domain exists at the limit if the center design is validated. The number of required tests for validation will increase in this case, considerably. For simplicity, the size of the local domains is fixed in the present work. Note also that the PPCA method (Section 3) addresses the inherent uncertainty in test results. The CAE model is deterministic. The calibration of the CAE model is done at the mean because of the variability in the test results.

\section{CONFIDENCE QUANTIFICATION}

The quantification of confidence that the design will perform as predicted once produced is an integral part of the proposed method. We utilize a model validation technique to compute that confidence.

Model validation is the process of comparing model outputs with experimental observations in order to assess the validity or predictive accuracy of computer models. Developing quantitative methods for model validation under uncertainty has attracted considerable research interest in recent years. Detailed discussion of model validation concepts can be found in many research articles [2-20].

For time-dependent systems, as the example considered in this paper, time histories of the prediction model and the test must be compared effectively, considering the data correlation among multiple time responses, and the uncertainties in both test and model prediction. Multiple independent univariate comparisons, without considering the correlation among different responses, may result in conflicting inferences on the model validity. A feature extraction, such as principal component analysis, is common in order to reduce the dimensionality and improve the efficiency and accuracy of the model validation process [21].

Bayesian methods have been developed to determine the predictive capabilities of computer models [8-13, 17, 18]. In this work, we adopt the Bayesian approach to quantify confidence reported in $[10-13,19]$ because i) it can incorporate additional information, including the modeler's belief, through the prior distribution of the model parameters, and ii) it can easily incorporate error and uncertainties associated with model predictions and experimental observations.

Specifically, a confidence metric is used to assess model predictions for time-dependent systems through multivariate validation. The metric provides a rational way to account for uncertainty and correlation of multiple responses, and is derived using Probabilistic Principal Component Analysis (PPCA) [22, 23] and interval-based multivariate Bayesian hypothesis testing. PPCA addresses multivariate correlation, data uncertainty, and dimensionality reduction, and intervalbased multivariate Bayesian hypothesis testing provides a quantitative assessment of the quality of the CAE model using a Bayes factor. The following describe the required computations as implemented in [20]. Details can be found in the same reference and in [10-13, 19].

\subsection{Probabilistic Principal Component Analysis}

The following is a step-by-step procedure on how to conduct a probabilistic principal components analysis [20]:

Step 1: Obtain multivariate test data $\mathbf{t} \in \mathfrak{R}^{m \times n}$; $\mathbf{t}=\left\{\mathbf{t}_{1}, \cdots, \mathbf{t}_{n}\right\}$, where $\mathbf{t}_{i} \in \mathfrak{R}^{m \times 1}$ and $m$ and $n$ are the number of test responses and the number of observations per response. Tipping and Bishop [22] assumed that

$$
\mathbf{t}_{i}=\mathbf{W} \mathbf{x}_{t_{i}}+\boldsymbol{\mu}_{t}+\boldsymbol{\varepsilon}, \quad i=1, \ldots, n
$$

where $\mathbf{W} \in \mathfrak{R}^{m \times p}$ is the PPCA transformation matrix, $\mathbf{x}_{t_{i}} \in \mathfrak{R}^{p \times 1}, i=1, \ldots, n$ is a vector of $p \leq m$ latent variables which cannot be observed, $\boldsymbol{\mu}_{t} \in \mathfrak{R}^{m \times 1}$ is the sample mean given by

$$
\boldsymbol{\mu}_{t}=\frac{1}{n} \sum_{i=1}^{n} \mathbf{t}_{i}
$$

and $\boldsymbol{\varepsilon} \in \mathfrak{R}^{m \times 1}$ is an isotropic Gaussian error with $\boldsymbol{\Sigma}_{t} \in \mathfrak{R}^{p \times p}$.

Step 2: Calculate the sample mean $\boldsymbol{\mu}_{t} \in \mathfrak{R}^{m \times 1}$ and covariance matrix $\mathbf{Q} \in \mathfrak{R}^{m \times m}$ of the test data using Equations (4) and (5)

$$
\mathbf{Q}=\frac{1}{n} \sum_{i=1}^{n}\left(\mathbf{t}_{i}-\boldsymbol{\mu}_{t}\right)\left(\mathbf{t}_{i}-\boldsymbol{\mu}_{t}\right)^{T}
$$

Step 3: Find the eigenvalues $\lambda_{1}, \ldots, \lambda_{m}$ in decreasing order, and the corresponding eigenvectors $\mathbf{u}_{1}, \ldots, \mathbf{u}_{m}$ of the covariance matrix $\mathbf{Q}$, where $\mathbf{u}_{j} \in \mathfrak{R}^{m \times 1}, j=1, \ldots, m$.

Step 4: For the chosen number $p$ of retained principal components, find estimates of the test variability $\hat{\sigma}_{t}^{2}$ and the transformation matrix $\hat{\mathbf{W}}$ using the equations

$$
\hat{\sigma}_{t}^{2}=\frac{1}{m-p} \sum_{j=p+1}^{m} \lambda_{j}
$$

and

$$
\hat{\mathbf{W}}=\mathbf{U}\left(\mathbf{K}-\hat{\sigma}_{t}^{2} \mathbf{I}\right)^{\frac{1}{2}}
$$

where $\mathbf{K} \in \mathfrak{R}^{p \times p}$ is a diagonal matrix with the eigenvalues $\lambda_{1}, \ldots, \lambda_{p}$ corresponding to the $p$ eigenvectors in $\mathbf{U}$. 
Step 5: Construct the $p$-dimensional reduced test data $\mathbf{r}_{t} \in \mathfrak{R}^{p \times n}$ with uncertainty $\boldsymbol{\Sigma}_{t} \in \mathfrak{R}^{p \times p}$ using the following Equations (8) and (9)

$$
\begin{gathered}
\mathbf{r}_{t_{i}}=\mathbf{M}^{-1} \hat{\mathbf{W}}^{T}\left(\mathbf{t}_{i}-\boldsymbol{\mu}_{t}\right), i=1, \ldots, n \\
\boldsymbol{\Sigma}_{t}=\hat{\sigma}_{t}^{2} \mathbf{M}^{-1}
\end{gathered}
$$

where

$$
\mathbf{M}=\mathbf{W}^{T} \mathbf{W}+\hat{\sigma}_{t}^{2} \mathbf{I}, \mathbf{M} \in \mathfrak{R}^{p \times p} .
$$

Step 6: Obtain multivariate CAE data $\mathbf{c} \in \mathfrak{R}^{m \times n}$ where $\mathbf{c}=\left\{\mathbf{c}_{1}, \cdots, \quad \mathbf{c}_{n}\right\}$ and $c_{i} \in \mathfrak{R}^{m \times 1}, i=1, \ldots, n$.

Step 7: Calculate the sample mean $\boldsymbol{\mu}_{c} \in \mathfrak{R}^{m \times 1}$ of the CAE data as

$$
\boldsymbol{\mu}_{c}=\frac{1}{n} \sum_{i=1}^{n} \mathbf{c}_{i} .
$$

Step 8: Use the same rotation matrix $\mathbf{M}^{-1} \hat{\mathbf{W}}^{T}$ (see Equation 8) to construct the $p$-dimensional reduced CAE data $\mathbf{r}_{c} \in \mathfrak{R}^{p \times n}$ from

$$
\mathbf{r}_{c_{i}}=\mathbf{M}^{-1} \hat{\mathbf{W}}^{T}\left(\mathbf{c}_{i}-\boldsymbol{\mu}_{t}\right), \quad \mathbf{r}_{c_{i}} \in \mathfrak{R}^{p \times 1}, \quad i=1, \ldots, n
$$

with uncertainty

$$
\boldsymbol{\Sigma}_{c}=\hat{\sigma}_{c}^{2} \mathbf{M}^{-1}, \quad \boldsymbol{\Sigma}_{c} \in \mathfrak{R}^{p \times p} .
$$

The estimate $\hat{\sigma}_{c}^{2}$ of the CAE variability is calculated from

$$
\hat{\sigma}_{c}^{2}=\frac{1}{m-p} \sum_{j=p+1}^{m} \lambda_{c_{j}}
$$

where $\lambda_{c_{1}}, \ldots, \lambda_{c_{m}}$ are the eigenvalues of the CAE data covariance matrix

$$
\mathbf{Q}_{c}=\frac{1}{n} \sum_{i=1}^{n}\left(\mathbf{c}_{i}-\boldsymbol{\mu}_{c}\right)\left(\mathbf{c}_{i}-\boldsymbol{\mu}_{c}\right)^{T}, \mathbf{Q}_{c} \in \mathfrak{R}^{m \times m} .
$$

\subsection{Interval-Based Bayesian Hypothesis Testing}

An interval-based multivariate Bayesian hypothesis testing method is used to compute a confidence value which quantitatively assesses the validity of the CAE model considering uncertainty. The following is a step-by-step procedure:

Step 1: Compute the difference between the reduced test and CAE data $\mathbf{S}=\left[\mathbf{s}_{1}, \ldots, \mathbf{s}_{n}\right]$ using Equations (8), (12) and (16)

$$
\mathbf{s}_{i}=\mathbf{r}_{t_{i}}-\mathbf{r}_{c_{i}}, \quad \mathbf{s}_{i} \in \mathfrak{R}^{p \times 1}, i=1, \ldots, n
$$

Step 2: Estimate the sample mean $\overline{\mathbf{S}}$ from

$$
\overline{\mathbf{S}}=\frac{1}{n} \sum_{i=1}^{n} \mathbf{s}_{i}
$$

and the covariance matrix $\boldsymbol{\Sigma} \in \mathfrak{R}^{p \times p}$ of $\mathbf{S}$ from Equations (9), (13) and (18)

$$
\boldsymbol{\Sigma}=\boldsymbol{\Sigma}_{t}+\boldsymbol{\Sigma}_{c}+\boldsymbol{\Sigma}_{s}
$$

where

$$
\boldsymbol{\Sigma}_{s}=\frac{1}{n} \sum_{i=1}^{n}\left(\mathbf{s}_{i}-\overline{\mathbf{S}}\right)\left(\mathbf{s}_{i}-\overline{\mathbf{S}}\right)^{T}, \quad \boldsymbol{\Sigma}_{s} \in \mathfrak{R}^{p \times p}
$$

Step 3: Assume that the prior information for $\boldsymbol{\mu}=\mathbf{E}\left(\mathbf{s}_{i}\right)$, $\boldsymbol{\mu} \in \mathfrak{R}^{p \times 1}$ is a multivariate normal distribution which is parameterized as $N_{p}(\boldsymbol{\rho}, \boldsymbol{\Lambda})$. The interval-based hypotheses are represented as $H_{o}:|\boldsymbol{\mu}| \leq \boldsymbol{\varepsilon}$ (accept the CAE model) versus $H_{a}:|\boldsymbol{\mu}|>\boldsymbol{\varepsilon}$ (reject the CAE model), where $\boldsymbol{\varepsilon}$ is a predefined threshold vector [12].

Obtain a prior distribution for $\boldsymbol{\mu} \sim N_{p}(\boldsymbol{\rho}, \boldsymbol{\Lambda})$. In the absence of a prior distribution, assume $\boldsymbol{\rho}=\mathbf{0}^{T}$ and $\Lambda=\Sigma$.

Step 4: Estimate the posterior distribution of $\boldsymbol{\mu}, \boldsymbol{\mu} \mid \mathbf{S} \sim N_{p}\left(\boldsymbol{\rho}_{o}, \boldsymbol{\Lambda}_{o}\right)$ using the following equations [24]

$$
\begin{gathered}
\boldsymbol{\rho}_{o}=\left(n \boldsymbol{\Sigma}^{-1}+\boldsymbol{\Lambda}^{-1}\right)^{-1}\left(n \boldsymbol{\Sigma}^{-1} \overline{\mathbf{S}}+\boldsymbol{\Lambda}^{-1} \boldsymbol{\rho}\right) \\
\boldsymbol{\Lambda}_{o}=\left(n \boldsymbol{\Sigma}^{-1}+\boldsymbol{\Lambda}^{-1}\right)^{-1} .
\end{gathered}
$$

Step 5: For a chosen threshold $\boldsymbol{\varepsilon}$, obtain the value of $K$ using the following equation

$$
K=\int_{-\varepsilon}^{\varepsilon} \frac{1}{(2 \pi)^{\frac{p}{2}}} \frac{1}{\sqrt{\left|\boldsymbol{\Lambda}_{o}\right|}} \exp \left(-\frac{1}{2}\left(\boldsymbol{\mu}-\boldsymbol{\rho}_{o}\right)^{T} \boldsymbol{\Lambda}_{o}^{-1}\left(\boldsymbol{\mu}-\boldsymbol{\rho}_{o}\right)\right) d \boldsymbol{\mu} .
$$

Step 6: Compute the following Bayes factor

$$
B_{M}=\frac{K}{1-K} \text {. }
$$

Step 7: Compute the confidence $C$ of accepting the model as

$$
C=P\left(H_{o} \mid \mathbf{S}\right)=\frac{B_{M} P\left(H_{o}\right)}{B_{M} P\left(H_{o}\right)+1-P\left(H_{o}\right)}
$$

if expert opinion $P\left(H_{o}\right)$ is available or as

$$
C=\frac{B_{M}}{B_{M}+1}
$$


if there is no expert opinion.

\section{A VIBRATORY BEAM EXAMPLE}

The vibratory response of the cantilever beam structure of Figure 2 is considered. The beam has a uniform rectangular cross section with width $W$, height $H$, and length $L=1 \mathrm{~m}$. The material Young modulus and density are $E=200 \mathrm{GPa}$ and $\rho$ $=7850 \mathrm{Kg} / \mathrm{m}^{3}$, respectively. The only load on the beam is a vertical force $f(t)$ at the tip.

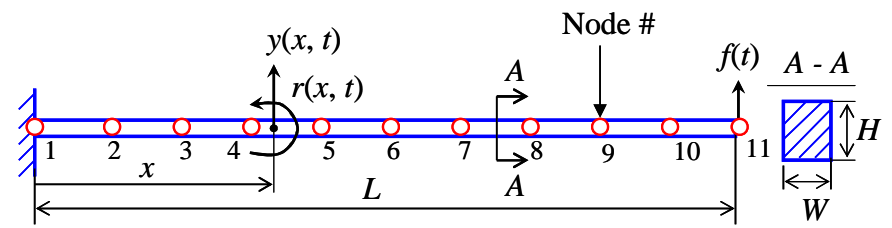

Figure 2. Cantilever beam of rectangular cross-section with tip point load $f(t)$

The vibratory displacement $y(x, t)$ in the vertical plane $x y$ satisfies the following partial differential equation under the Euler-Bernouli beam [25] assumption

$$
\rho A \frac{\partial^{2} y}{\partial t^{2}}+E I \frac{\partial^{4} y}{\partial x^{4}}=0
$$

where $I$ is the area moment of inertia. Equation (26) is solved using the two initial conditions $y(x, 0)=0$ and $\dot{y}(x, 0)=0$; (zero initial displacement and zero initial velocity), and four boundary conditions (two at each end) indicating that the left end of the beam is fixed (displacement and rotation are equal to zero) and the right end is free (bending moment is equal to zero and shear force is equal to the applied tip force).

The finite-element method is used to calculate numerically the forced response. The beam is discretized using $N_{E}$ elements of equal length $L_{B}=L / N_{E}$, resulting in $N=N_{E}+1$ equally spaced nodes as shown in the Figure 2 . The coordinate $x$ of the $i^{t h}$ node is $x_{i}=(i-1) L_{B}$. The following timedependent point load is applied on the tip of the beam

$$
f(t)=\sin (48 t)+\sin (130 t) \mathrm{N} .
$$

Using the finite-element method, the differential Equation (26) results in the following equations of motion in discretized form

$$
[M] \ddot{\mathbf{y}}+[C] \dot{\mathbf{y}}+[K] \mathbf{y}=\mathbf{f}(t)
$$

where $[M],[C]$, and $[K]$ the mass, damping, and stiffness matrices, $\mathbf{y}$ is the vector of nodal responses, and $\mathbf{f}$ is the vector of nodal forces. There are two degrees of freedom at each node; the vertical displacement $y(x, t)$, and the rotation $r(x, t)=\partial y(x, t) / \partial x$. The vector $\mathbf{y}$ has therefore, $2 N$ components where $N$ is the number of nodes.

The forced response is calculated using Newmark's time integration method [25] for $0 \leq t \leq 2 \mathrm{sec}$ with a time step of
$\Delta t=0.001 \mathrm{sec}$. We assume that there is structural damping so that

$$
[C]=\alpha[M]+\beta[K]
$$

where $\alpha=0.003$ and $\beta=0.003$.

\subsection{Design Optimization Problem}

The following optimization problem is solved

$$
\begin{array}{cc}
\qquad \min _{\mathbf{d}} \operatorname{Mass}(\mathbf{d})=\rho d_{1} d_{2} L \\
\text { where } \mathbf{d}=\left\{\begin{array}{ll}
d_{1} & d_{2}
\end{array}\right\}=\left\{\begin{array}{ll}
W & H
\end{array}\right\} \\
\text { s.t. } \quad \max _{t}|y(\mathbf{d}, t)| \leq y^{t} \\
\max _{t}|r(\mathbf{d}, t)| \leq r^{t} \\
\underline{\mathbf{d}} \leq \mathbf{d} \leq \overline{\mathbf{d}} .
\end{array}
$$

The beam mass is minimized considering the width $W$ and height $H$ of the beam cross section as design variables. The maximum absolute displacement and rotation of the beam tip for $t \in\left[\begin{array}{ll}0,2 & 2\end{array} \mathrm{sec}\right.$ must be less or equal to $y^{t}=0.001 \mathrm{~m}$ and $r^{t}=0.001$ radians, respectively. The design domain is defined by the lower and upper bounds $\underline{\mathbf{d}}$ and $\overline{\mathbf{d}}$ of $\mathbf{d}$, where $\underline{\mathbf{d}}=\left(\begin{array}{ll}\underline{d}_{1} & \underline{d}_{2}\end{array}\right)=\left(\begin{array}{ll}0.015 & 0.005\end{array}\right) \mathrm{m}$ and $\overline{\mathbf{d}}=\left(\begin{array}{ll}\bar{d}_{1} & \bar{d}_{2}\end{array}\right)=$ $\left(\begin{array}{ll}0.05 & 0.05\end{array}\right) \mathrm{m}$.

\subsection{Definition of Test}

To obtain the test response (displacement and rotation), we assume that the fixed boundary condition at the left end of the beam (Figure 3) can not be perfectly achieved. This is simulated by using a smaller cross-sectional area for the first beam element with a width and height equal to $W_{1}=W-0.002 \mathrm{~m}$ and $H_{1}=H-0.002 \mathrm{~m}$ respectively, where $W$ and $H$ are the cross-sectional dimensions for all other beam elements. This is practically equivalent to having a smaller rotational stiffness at the root of the beam which becomes substantially small at the low bound values $\left(\begin{array}{ll}\underline{d}_{1} & \underline{d}_{2}\end{array}\right)=\left(\begin{array}{ll}\underline{W} & \underline{H}\end{array}\right)=\left(\begin{array}{ll}0.015 & 0.005\end{array}\right) \mathrm{m}$. This "experimental inaccuracy" is assumed unknown in building the CAE model.

For each test specimen, we also consider a small manufacturing error for each component of the vector $\mathbf{R}=\left\{\begin{array}{lllllll}L & W & H & E & \rho & \alpha & \beta\end{array}\right\}$. The error is $\Delta \mathbf{R}=\left\{\begin{array}{lllllll}\Delta L & \Delta W & \Delta H & \Delta E & \Delta \rho & \Delta \alpha & \Delta \beta\end{array}=\right.$

$\left\{\begin{array}{lllllll}0.0005 & 0.0002 & 0.0004 & 10^{4} & 100 & 0.001 & 0.001\end{array}\right\}$ with all units according to the metric system. For each test we assume that $\mathbf{R}=\mathbf{R}_{0}+\Delta \mathbf{R}^{*}$ where $\mathbf{R}_{0}$ is the nominal value and each component of $\Delta \mathbf{R}^{*}$ is normally distributed $\left(\Delta \mathbf{R}^{*} \sim N\left(\boldsymbol{\mu}, \boldsymbol{\sigma}^{2}\right)\right)$ with $\boldsymbol{\mu}=[\Delta \mathbf{R}+(-\Delta \mathbf{R})] / 2=0$ and $\boldsymbol{\sigma}=[\Delta \mathbf{R}-(-\Delta \mathbf{R})] / 6$. Every time a test is conducted, a sample value of $\Delta \mathbf{R}^{*}$ is drawn from its normal distribution and used to calculate $\mathbf{R}=\mathbf{R}_{0}+\Delta \mathbf{R}^{*}$. 
To simulate the test results, Equation (3) is solved numerically for $y(x, t)$ and $r(x, t)$ using $N_{E}=10$ beam elements, the test configuration of Figure 3 , and the manufacturing error. A measurement error is also considered using the Gaussian white noise $N\left(0,1^{2}\right)$ modulated by the factor $0.05 \cdot \max y(t) \mathrm{m}$ for $t \in\left[\begin{array}{ll}0 & 2\end{array}\right] \mathrm{sec}$ so that the test response is

$$
y_{T E S T}(x, t)=y(x, t)+0.06 \cdot \max _{t \in\left[\begin{array}{ll}
0 & 2
\end{array}\right]} y(t) \cdot N\left(0,1^{2}\right) \mathrm{m} .
$$

A similar expression holds for $r_{\text {TEST }}(x, t)$.

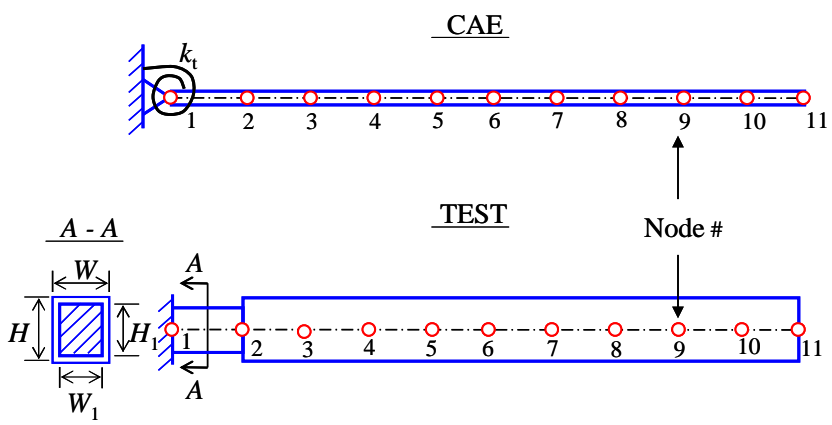

Figure 3. Cantilever beam of rectangular cross-section for test and CAE cases; the test uses a reduced cross-section close to the fixed end and the CAE model assumes a pinned left end with a rotational spring constant $k_{\mathrm{t}}$

\subsection{Description of CAE Model}

The CAE model is of uniform cross-section with dimensions $W$ and $H$ (Figure 3 ). Because the correct rotational boundary condition of the test is not known, we use a rotational spring $k_{t}$ which is calibrated during the validation process. The calibration parameter vector $\mathbf{p}=$ $\left\{\begin{array}{llllll}k_{t} & W & H & E & \rho & \beta\end{array}\right\} \quad$ (Section 2) is defined as $\mathbf{p}=\mathbf{p}_{0}+\Delta \mathbf{p}$, where $\mathbf{p}_{0}$ is the nominal vector that is unchanged during calibration, and $\Delta \mathbf{p}=$ $\left\{\Delta k_{t} \quad \Delta W \quad \Delta H \quad \Delta E \quad \Delta \rho \quad \Delta \beta\right\}$ is the variable part with $\Delta k_{t} \in\left[\begin{array}{ll}k_{t_{0}} & 100 \cdot k_{t_{0}}\end{array}\right], \Delta W \in\left[\begin{array}{ll}-0.0002 & 0.0002\end{array}\right], \Delta H \in$ $\left[\begin{array}{ll}-0.0004 & 0.0004\end{array}\right], \Delta E \in\left[\begin{array}{ll}-10^{4} & 10^{4}\end{array}\right], \Delta \rho \in\left[\begin{array}{ll}-100 & 100\end{array}\right]$, and $\Delta \beta \in\left[\begin{array}{ll}-0.001 & 0.001\end{array}\right]$. The nominal rotational stiffness $k_{t_{0}}$ is equal to the beam element rotational stiffness $4 E I / L_{B}$.

Equation (26) is solved numerically for $y(x, t)$ and $r(x, t)$ using $N_{E}=10$ beam elements so that

$$
y_{C A E}(x, t)=y(x, t) \text { and } r_{C A E}(x, t)=r(x, t) .
$$

The response of the CAE model is deterministic with no manufacturing error or noise.

\subsection{Results and Discussion}

Figure 4 shows how the confidence $C$ varies with the design variables $d_{1}$ and $d_{2}$ before the CAE model is calibrated, and after it is calibrated at the initial design (0.024,
0.042). Before calibration, the confidence exhibits a highly nonlinear behavior with large variations between low and high values of approximately $40 \%$ and $80 \%$, respectively. After calibration, the confidence is almost the same with the noncalibrated case for high $d_{2}$ values and close to $100 \%$ for low $d_{2}$ values.

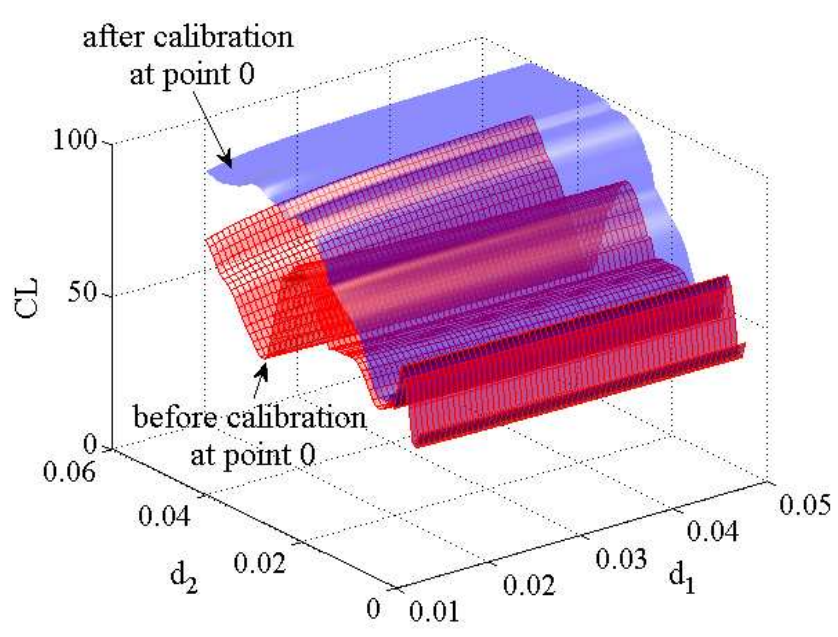

Figure 4. Confidence value as a function of design variables $d_{1}$ and $d_{2}$

Two principal components $(p=2)$ were retained. It was found that the confidence value did not change for $p$ greater than two.

Figures 5 and 6 compare the tip deflection between test and CAE at the initial design $(0.024,0.042)$ before and after model validation through calibration. The $C$ value improves from $38.8 \%$ to $99.3 \%$.

Figures $7 \mathrm{a}$ and $7 \mathrm{~b}$ show the optimization process in the design space for the non-calibrated and calibrated cases, respectively. In the former, the $\mathrm{CAE}$ model is not calibrated during the process while in the latter the CAE model is calibrated according to the methodology of Section 2.

The figures show the displacement constraint before calibration (non-calibrated case), after calibration at point 0 (initial design $(0.024,0.042) \mathrm{m}$ ), and after calibration at point 4 (design $(0.0167,0.0285) \mathrm{m}$ before final optimum). The equal objective value lines are also shown. The mass objective reduces towards the low left corner where both $d_{1}$ and $d_{2}$ reduce simultaneously. The displacement constraint dominates the rotation constraint in the entire design space. Also, we have There is a local optimal design at $(0.015,0.0395) \mathrm{m}$ indicated by point 4 in Figure $7 \mathrm{a}$, and another local optimal design at $(0.0167,0.0276) \mathrm{m}$ indicated by point 5 in Figure $7 \mathrm{~b}$.

The same initial design of $(0.024,0.042)$ is used in both cases. The sequence of local domains from the initial design to the optimal design is shown for the non-calibrated and calibrated CAE models. An increasing order numbering, starting from zero (initial design), indicates the center location of each local domain. For the calibrated CAE case, a cross is used whenever the design is validated; i.e. the CAE model is calibrated by maximizing the $C$ value (Section 2 ) if the initial $C$ value is below the threshold $C^{t}$. 


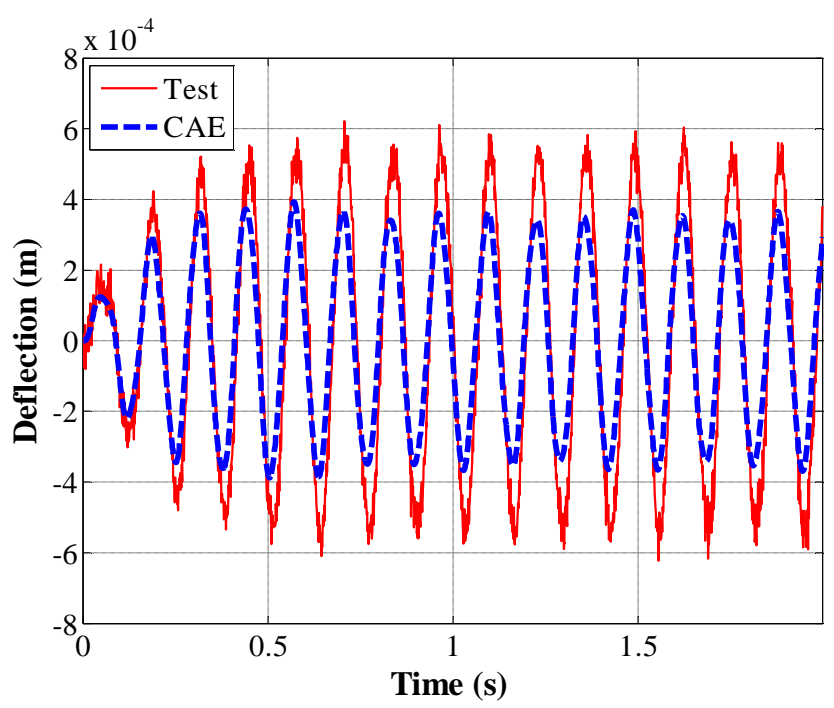

Figure 5. Comparison of tip displacement between test and $\mathrm{CAE}$ at initial design before calibration

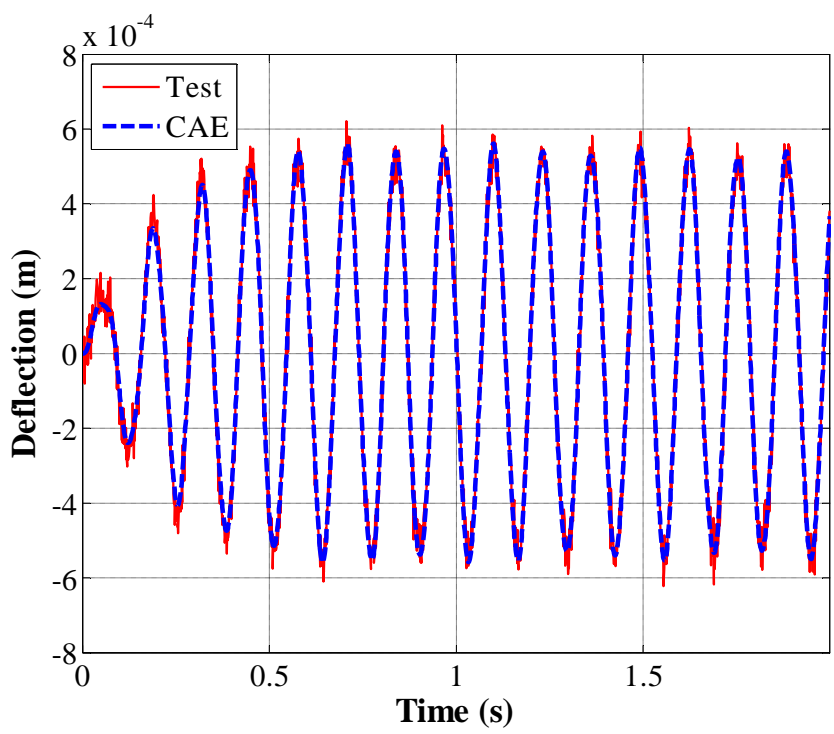

Figure 6. Comparison of tip displacement between test and $\mathrm{CAE}$ at initial design after calibration

Figure $7 \mathrm{~b}$ shows the displacement constraint before and after calibration at the initial design (point 0 ). Because of the difference between the two lines, the optimization converges to the local optimum $(0.015,0.0395) \mathrm{m}$ for the non-calibrated CAE model and to the local optimum $(0.0167,0.0276) \mathrm{m}$ for the calibrated CAE model. This indicates the significance of calibrating the CAE model during the optimization process.

Figure 8 shows the design history for the non-calibrated (dotted line) and the calibrated (solid line) CAE model. Both histories start from the same initial design and reach the same intermediate optimum for the initial local domain. Subsequently, the optimization paths divert due to different CAE models because of calibration at the center design $\mathbf{d}_{0}=\left(\begin{array}{ll}0.024 & 0.042\end{array}\right) \mathrm{m}$ of local domain 0 for the calibrated case.

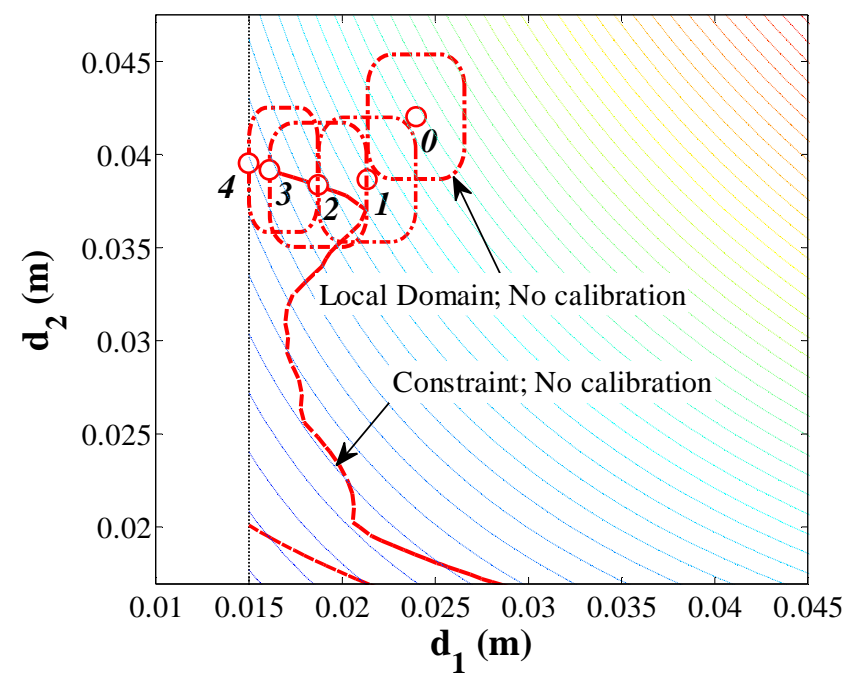

(a)

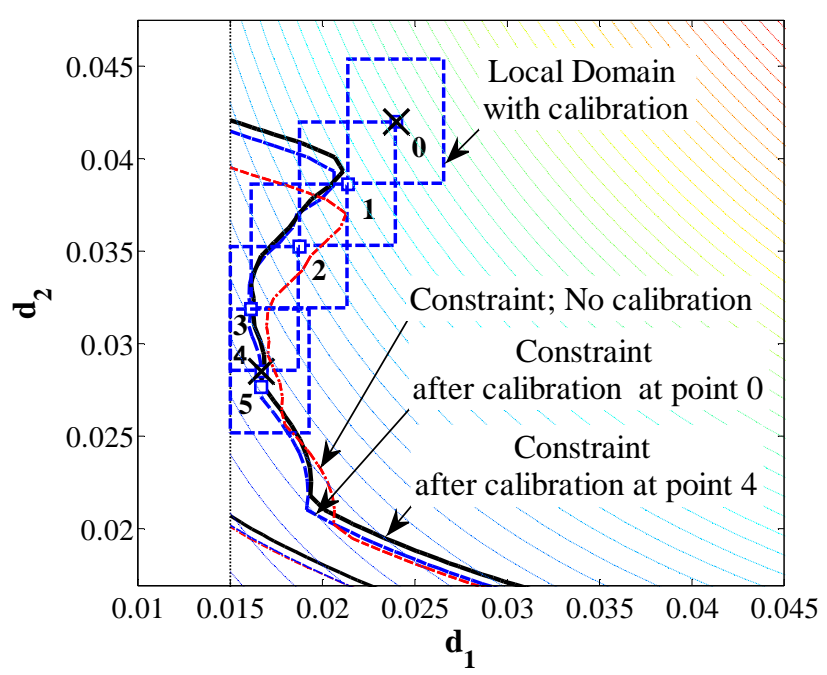

(b)

Figure 7. Optimization process in the design space for non-calibrated and calibrated CAE model

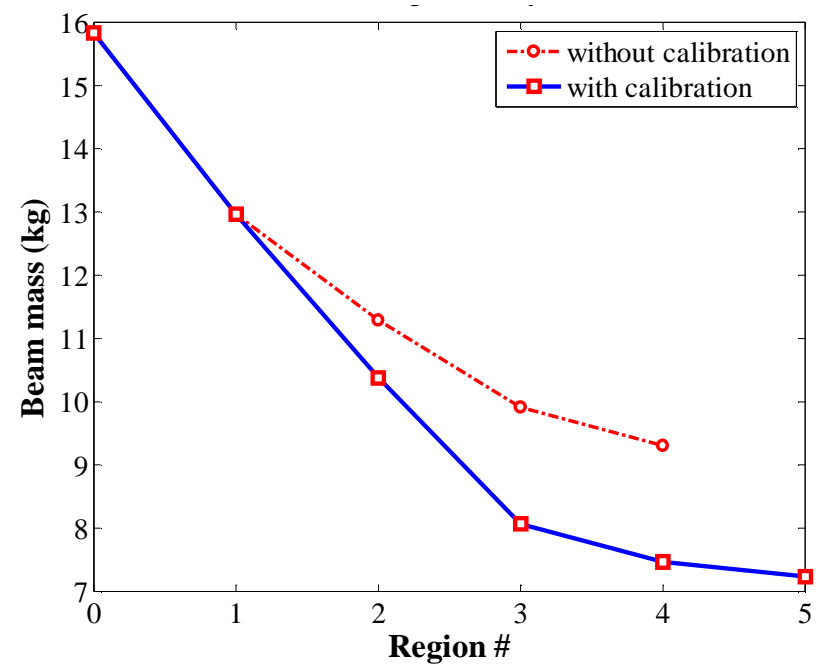

Figure 8. Design optimization history for non-calibrated and calibrated CAE model 
Table 1 shows the results at the center of each local domain. The values of the design variables are shown in columns 2 and 3. Column 4 shows the objective function and columns 5 and 6 show the value of the two constraints. The last column lists the value of the confidence level. The displacement constraint becomes active at local domains 2, 3, and 4 as the maximum displacement $y^{\max }$ at the tip of the beam is equal to the target $y^{t}=0.001 \mathrm{~m}$. As expected, the confidence value $C$ is small for all local domains since the CAE model is not calibrated.

Table 1. Optimization details for non-calibrated CAE case

\begin{tabular}{|c|c|c|c|c|c|c|}
\hline Domain \# & $\begin{array}{c}\boldsymbol{W} \\
(\mathbf{m})\end{array}$ & $\begin{array}{c}\boldsymbol{H} \\
(\mathbf{m})\end{array}$ & $\begin{array}{c}\text { Mass } \\
(\mathbf{k g})\end{array}$ & $\begin{array}{c}\max (\boldsymbol{y}) \\
(\mathbf{m})\end{array}$ & $\begin{array}{c}\max (\boldsymbol{r}) \\
(\mathbf{r a d})\end{array}$ & $\begin{array}{c}\boldsymbol{C} \\
(\boldsymbol{\%})\end{array}$ \\
\hline 0 & 0.024 & 0.042 & 15.8256 & 0.0004 & 0.0003 & 38.8 \\
\hline 1 & 0.0214 & 0.0386 & 12.9621 & 0.0008 & 0.0006 & 27.7 \\
\hline 2 & 0.0188 & 0.0383 & 11.2867 & 0.001 & 0.0007 & 27.3 \\
\hline 3 & 0.0161 & 0.0391 & 9.9107 & 0.001 & 0.0007 & 23.8 \\
\hline 4 & 0.0150 & 0.0395 & 9.3048 & 0.001 & 0.0007 & 21.2 \\
\hline
\end{tabular}

Table 2 shows the results for the calibrated CAE case. At the initial design (center of local domain 0 ) the $C$ value is equal to $38.8 \%$ and $99.3 \%$ before and after model validation through calibration, respectively. The CAE model stays valid in local domains 1,2 , and 3 since the $C$ value is above the threshold $C^{t}=80 \%$. In the final local domain 4 , the CAE model was re-calibrated because the value of $C=77.9 \%$ fell below the target; the final $C$ value was maximized to $85.1 \%$. The final optimum of $(0.0167,0.0276) \mathrm{m}$ was obtained using the lastly updated CAE model with $C=85.1 \%$. The displacement constraint became active for the designs corresponding to local domains 1, 3, 4, and 5. As shown in Figure $7 b$, these designs are in the infeasible domain of the non-calibrated case. This indicates that after each calibration of the CAE model, the optimization constraint boundaries change. Figure $7 \mathrm{~b}$ also shows the constraint boundary for the final calibrated CAE model.

Table 2. Optimization details for calibrated CAE case

\begin{tabular}{|c|c|c|c|c|c|c|}
\hline Domain \# & $\begin{array}{c}\boldsymbol{W} \\
(\mathbf{m})\end{array}$ & $\begin{array}{c}\boldsymbol{H} \\
(\mathbf{m})\end{array}$ & $\begin{array}{c}\text { Mass } \\
(\mathbf{k g})\end{array}$ & $\begin{array}{c}\max (\boldsymbol{y}) \\
(\mathbf{m})\end{array}$ & $\begin{array}{c}\max (\boldsymbol{r}) \\
(\mathbf{r a d})\end{array}$ & $\begin{array}{c}\boldsymbol{C} \\
(\boldsymbol{\%})\end{array}$ \\
\hline 0 & 0.024 & 0.042 & 15.8256 & 0.000393 & 0.000270 & $\mathbf{3 8 . 8} /$ \\
\hline 1 & 0.0214 & 0.0386 & 12.9621 & 0.001 & 0.000642 & 98.3 \\
\hline 2 & 0.0188 & 0.0352 & 10.3767 & 0.000931 & 0.000603 & 84.14 \\
\hline 3 & 0.0161 & 0.0319 & 8.0696 & 0.001 & 0.000670 & 81.8 \\
\hline 4 & 0.0167 & 0.0285 & 7.4695 & 0.001 & 0.000636 & $\mathbf{7 7 . 9}$ \\
\hline 5 & 0.0167 & 0.0276 & 7.2364 & 0.001 & 0.000645 & $\mathbf{8 5 . 1}$ \\
\hline
\end{tabular}

Table 3 shows the values of all calibration parameters for the calibrated CAE model of each local domain. The CAE model was calibrated at the initial design and the $C$ value was increased from $38.8 \%$ to $99.3 \%$. The calibration parameters are $\Delta k_{t}=1.88 k_{t_{o}}, \Delta H=-1.16 \times 10^{-5} \mathrm{~m} \quad \Delta E=-0.16 \mathrm{GPa}$ $\Delta \rho=3.99 \mathrm{~kg} / \mathrm{m}^{3}$, and $\Delta \beta=1.59 \times 10^{-4}$. This calibrated CAE model was used in local domains $1,2,3$, and 4 . The CAE model was again calibrated at the fourth local domain increasing $\mathrm{C}$ from $77.9 \%$ to $85.1 \%$ and the final optimal design $\mathbf{d}=\left(\begin{array}{ll}0.0167 & 0.0276\end{array}\right) \mathrm{m}$ was obtained.

Table 3. Calibration details

\begin{tabular}{|c|c|c|c|c|c|c|}
\hline Domain \# & $\Delta k_{t} / k_{t_{o}}$ & $\begin{array}{c}\boldsymbol{\Delta H} \\
\left(\mathbf{1 0} \mathbf{H}^{-5} \mathbf{m}\right)\end{array}$ & $\begin{array}{c}\boldsymbol{\Delta E} \\
(\mathbf{G P a})\end{array}$ & $\begin{array}{c}\boldsymbol{\Delta \rho} \\
\left(\mathbf{k g} / \mathbf{m}^{3}\right)\end{array}$ & $\begin{array}{c}\boldsymbol{\Delta \beta} \\
\left(\mathbf{1 0}^{-4}\right)\end{array}$ & $\begin{array}{c}\boldsymbol{C} \\
(\boldsymbol{\%})\end{array}$ \\
\hline 0 (Before Cal.) & 0.0 & 0.0 & 0.0 & 0.0 & 0.0 & $\mathbf{3 8 . 8}$ \\
\hline 0 (After Cal.) & 1.88 & -1.16 & -0.16 & 3.99 & 1.59 & $\mathbf{9 9 . 3}$ \\
\hline 1 & 1.88 & -1.16 & -0.16 & 3.99 & 1.59 & 98.3 \\
\hline 2 & 1.88 & -1.16 & -0.16 & 3.99 & 1.59 & 84.14 \\
\hline 3 & 1.88 & -1.16 & -0.16 & 3.99 & 1.59 & 81.8 \\
\hline 4 (Before Cal.) & 1.88 & -1.16 & -0.16 & 3.99 & 1.59 & $\mathbf{7 7 . 9}$ \\
\hline 4 (After Cal.) & 1.82 & -36.0 & -3.2 & 100.0 & 1.47 & $\mathbf{8 5 . 1}$ \\
\hline
\end{tabular}

The optimization process with the calibrated CAE model followed a different path and converged to a better optimum. This example demonstrates the value of validating designs during the optimization process.

\section{SUMMARY AND CONCLUSIONS}

We presented a methodology for validating designs as they are generated during the simulation-based optimization process, emphasizing that the validation should be on designs, the end goals of optimization, rather than the computational models used to obtain them. The presented methodology requires the validity of the models only at designs generated during a sequential optimization approach where suboptimization problems are solved within local design domains that are subsets of the entire domain. This is different from the current practice where a-priori validation of simulation models is performed throughout the entire design space.

The methodology uses a quantitative confidence metric based on PPCA to address multivariate correlation, data uncertainty, and dimensionality reduction, and interval-based multivariate Bayesian hypothesis testing using a Bayes factor.

The vibratory response of a cantilever beam was used to illustrate the process. We showed that by calibrating the CAE model when necessary, the optimizer followed a different path which led to an optimum different from that obtained using a non-calibrated CAE model.

The present work assumes that tests can be performed at any location of the design space. It also uses local domains of same size. Future work is underway to remove this restriction. A different method to calibrate the CAE model is also considered using parameter screening based on global sensitivities [26].

\section{ACKNOWLEDGMENT}

Funding was provided by the Automotive Research Center (ARC), a U.S. Army Center of Excellence in Modeling and Simulation of Ground Vehicles at the University of Michigan. The support is gratefully acknowledged. The support does not constitute an endorsement by the funding agency of the opinions expressed in the paper. 


\section{REFERENCES}

1. Booker, A. J., Dennis, Jr., J. E., Frank, P. D., Serafini, D. B., Torczon, V., and Trosset, M. W., "A Rigorous Framework by Surrogates for Optimization of Expensive Functions," Structural Optimization, 17, 1-13, 1999.

2. Bayarri, M. J., Berger, J. O., Paulo, R., Sacks, J., Cafeo, J. A., Cavendish, J., Lin, C. H., and Tu, J., "A Framework for Validation of Computer Models," Technical Report Number 162, National Institute of Statistical Sciences, Research Triangle Park, New Jersey, 2005.

3. Easterling, R. G., "Statistical Foundations for Model Validation," Technical Report SAND2003-0287, Sandia National Laboratories, Albuquerque, New Mexico, 2003.

4. Oberkampf, W. L., and Trucano, T. G., "Design of and Comparison with Verification and Validation Benchmarks," Sandia National Laboratories, Technical Report Sand. No 2006-5376C, Albuquerque, New Mexico, 2006.

5. Oberkampf, W. L., Trucano, T. G., and Hirsch, C., "Verification, Validation, and Predictive Capabilities in Computational Engineering and Physics," Sandia National Laboratories, Technical Report Sand. No 20033769, Albuquerque, New Mexico, 2003.

6. Oberkampf, W. L., Trucano, T. G., and Hirsch, C., "Verification, Validation, and Predictive Capability in Computational Engineering and Physics," Applied Mechanics Reviews, 57(5), 345-384, 2004.

7. Trucano, T. G., Easterling, R. G., Dowding, K. J., Paez, T. L., Urbina, A., Romero, V. J., Rutherford, B. M., and Hills, R. G., "Description of the Sandia Validation Metrics Project," Sandia National Laboratories, Technical Report Sand. No 2001-1339, Albuquerque, New Mexico, 2001.

8. Rebba, R., Model Validation and Design under Uncertainty, PhD Thesis, Vanderbilt University, 2005.

9. Rebba, R., and Mahadevan, S., "Model Predictive Capability Assessment under Uncertainty," AIAA Journal, 44(10), 2376-2384, 2006.

10. Jiang, X., and Mahadevan, S., "Bayesian risk-based decision method for model validation under uncertainty," Reliability Engineering and System Safety, 92(6), 707718, 2007.

11. Jiang, X., and Mahadevan, S., "Bayesian Validation Assessment of Multivariate Computational Models," Journal of Applied Statistics, 35(1), 49-65, 2008.

12. Jiang, X., and Mahadevan, S., "Bayesian Wavelet Method for Multivariate Model Assessment of Dynamical Systems," Journal of Sound and Vibration, 312(4-5), 694-712, 2008.

13. Jiang, X., and Mahadevan, S., "Bayesian Inference Method for Model Validation and Confidence
Extrapolation," Journal of Applied Statistics, 36(6), 659677, 2009.

14. Oberkampf, W. L., and Barone, M. F., "Measures of Agreement between Computation and Experiment: Validation Metrics," Journal of Computational Physics, 217(1), 5-36, 2006.

15. Hills, R. G., and Leslie, I., "Statistical Validation of Engineering and Scientific Models: Validation Experiments to Application," Sandia National Laboratories, Technical Report Sand. No 2003-0706, Albuquerque, New Mexico, 2003.

16. Mahadevan, S., and Rebba, R., "Validation of Reliability Computational Models using Bayes Networks," Reliability Engineering and System Safety, 87(2), 223232, 2005.

17. Chen, W., Baghdasaryan, L., Buranathiti, T., and Cao, J., "Model Validation via Uncertainty Propagation and Data Transformations," AIAA Journal, 42(7), 1406-1415, 2004.

18. Chen, W., Xiong, Y., Tsui, K. - L., and Wang, S., "A Design-Driven Validation Approach using Bayesian Prediction Models," Journal of Mechanical Design, 130(2), 021101-1-12, 2008.

19. Jiang, X., Yang, R. - J., Barbat, S., and Weerappuli, P., "Bayesian Probabilistic PCA Approach for Model Validation of Dynamic Systems," SAE International Journal of Materials \& Manufacturing, 2(1), 555-563, 2009.

20. Pai, Y., Investigation of Bayesian Model Validation Framework for Dynamic Systems, MS thesis, Department of Mechanical Engineering, University of Michigan, Ann Arbor, 2009.

21. Hemez, F. M., and Doebling, S. W., "Validation of Structural Dynamics Models at Los Alamos National Laboratory," Proceedings, 41st AIAA/ASME/ASCE/AHS/ASC Structures, Structural Dynamics and Materials Conference, Atlanta, GA, 2000.

22. Tipping, M. E., and Bishop, C. M., "Probabilistic Principal Component Analysis," Journal of the Royal Statistical Society: Series B (Statistical Methodology), 61(3), 611-622, 1999.

23. Joliffe, I. T., Principal Component Analysis, Springer, New York, 2002.

24. Gelman, A., Carlin, J. B., Stern, H. S., and Rubin, D. B., Bayesian Data Analysis, Taylor \& Francis, Inc., 85-86, 2003.

25. Rao, S. S., Mechanical Vibrations, $4^{\text {th }}$ Edition, Pearson Prentice Hall, 2004.

26. Drignei, D., Mourelatos, Z. P., and Rebba, R., "Parameter Screening in Dynamic Computer Model Calibration using Global Sensitivities," Proceedings, 2010 ASME IDETC/CIE, Paper DETC2010-28343, Montreal, Canada, 2010. 